\title{
The Assessment of Assistive Technology Outcomes, Effects and Costs
}

\author{
Gert Jan Gelderblom and Luc P. de Witte \\ $i R v$, Institute for Rehabilitation Research, P.O. Box 192, 6430 AD Hoensbroek, The Netherlands \\ Http:www.irv.nl; E-mail; gj.gelderblom@irv.nl
}

\section{Purpose of the special issue}

Due to the pressure to economize, increased consumerism and concerns about quality in relation to costs, the assessment of the outcome of assistive technology (AT) has been given increasing attention in recent years. The political and economical call for rationalising the amount of recourses spent on assistive technology, but also the intention to enhance the outcome of AT, make insight into the outcome of provided assistive technology necessary. The instruments and methods involved in the assessment of outcome are the topic of this special issue.

The prime objective of this special issue is to present a set of instruments that can be used to study the effects or costs of AT delivery. Through the provided standardised description the reader is enabled to judge the applicability to specific conditions and the soundness of each instrument in general. Because the discipline of AT outcome assessment is relatively young, only few instruments specifically designed to measure outcomes of AT and with demonstrated soundness are available, and the applicability of outcome instruments from adjacent fields of application is to be demonstrated anew. However, the application of a well developed instrument does not automatically guarantee appropriate assessment of AT outcomes. An instrument may not be suitable for a specific type of AT and its use, or the circumstances of application may vary considerably from those during its development, due to cultural and/or language variation. The set of instruments included in this issue covers most of the best ones presently available and should be considered for use when appropriate.

A second objective of this special issue is to stimulate the use of standardised instruments. To facilitate comparisons of outcomes of AT, either between users, AT alternatives or use conditions, the data to compare should preferably be obtained with the same instruments. Moreover, the application of standardized instruments may support the effect of AT use because a better informed selection can be made on the basis of the outcome assessment.

Finally, a special issue providing an overview of the state of the art may serve as an inspiration for further development of existing or new instruments as it outlines topics requiring further attention.

\section{Outcome assessment of Assistive Technology}

Assessing outcome can be required to demonstrate the efficacy of the application of new technology, to establish the effectiveness of assistive technology over time or to steer the development of new assistive technology [1].

Efficacy of an AT device is determined by the effect resulting from its use in comparison to the effect claimed beforehand. The nature of this effect can be manifold, depending on the user(-population), the device and the use conditions. Effectiveness on societal level is often considered in relation to costs; i.e., costeffectiveness. Then effectiveness is regarded as the 
whole of benefits potentially resulting from the introduction of any intervention. Benefits can be expressed in financial units, utility measures or outcomes. Costs on the other hand, are expressed in both financial and other terms, all following from the availability of the intervention. The cost-effectiveness of the intervention is then established by weighing the benefits against the costs.

When the benefits of the intervention can be expressed in financial units, obtaining a cost-benefit ratio is easy. When the benefits are expressed in a uniform utility measures, for example Quality Adjusted Life Years (QUALY), direct comparisons between benefit and costs are also possible by adding a financial value per unit of the utility measure. The value of such comparisons is however debated upon. When the benefits are expressed in outcomes, i.e., changes produced by the Assistive Technology in the lives of users and their environments [3, p. 34029], then comparisons between benefit and costs can no longer be made directly. In most fields of medicine, decisions on the application of medical interventions are evidence based by a direct comparison between benefit and costs, but the provision of AT has been an exception. One of the reasons for this exception is the complexity of AT outcomes. This complexity originates from a) the diversity in contributing variables, b) outcome being a multidimensional concept, c) the embedding of an AT device, and d) the goals to be reached with an assistive device.

a) The impact of AT does not only depend on the characteristics of the end user, but also on the context of application and the type of AT. Living conditions and/or type of care can influence the outcome. The diversity within assistive technology introduces a large spectrum of potential outcomes to be assessed by an instrument, e.g., the functional effect of a wheelchair is completely different from that of a computer.

b) The outcome of AT is at closer inspection a composition of various effects at different levels and of different nature. Effects on user satisfaction, functional independence, societal and individual gains, increased social participation, enhanced normative social roles, the promotion and sustaining of employment and facilitation of activities of daily living may all add to the outcome of AT.

c) Provision of AT is seldom an isolated action. Users commonly make use of one or more AT devices in combination with care and the outcome of a specific device should be assessed with reference to the context it is embedded in. d) The goals for providing a device can be divers and highly individual. Between persons an assistive device can be provided to reach different goals and reaching an identical goal may require different devices for different persons.

Instruments (or a set of instruments) assessing the outcome of assistive technology should therefore be generic and flexible, consider the range of possible outcome elements with reference to the context the device is embedded in and express the effect in terms of the extent to which individual goals were reached.

\section{Selection of instruments for outcome assessment of AT}

In light of the described importance of AT outcome assessment and the complexity related to the nature of AT outcomes, the methods and instruments applied in the assessment of AT outcomes should be given appropriate attention. The quality of an instrument affects the quality of the data obtained with the instrument. Therefore it is important to know how valid, reliable and feasible an instrument is.

The outcome of AT can be measured at different conceptual levels ranging from functional performance to quality of life. There is a relatively small number of instruments specifically designed for the assessment of AT outcome. These instruments all aim at a specific type of outcome of the application of AT. However, the outcome of AT is a composition of various effects; facilitation of activities of daily living, change in functional independence, user satisfaction, societal and individual gains, effects on participation; employment and social roles. Therefore, establishing the effect of AT may require more than one instrument depending on the type of question underlying the assessment of outcomes.

Besides the assessment of outcomes also the assessment of costs resulting from AT application can involve a range of variables. Methods for assessing costs in general may be provided by the Health Technology Assessment (HTA) discipline, but the application of these methods to AT provision and use can be troublesome. There are only very few instruments available providing a tailored method of establishing the costs involved with the provision of AT.

The eight instruments included in this special issue comprise a cross section of the available instruments in the field. All are generic, i.e., designed for the evalua- 
Table 1

Overview of instruments in this issue and the concepts they address

\begin{tabular}{ll}
\hline Instrument & Concept \\
\hline EuroQol and PIRS & Quality of Life \\
IPPA & Individual Goals \\
Life-H & Social Participation \\
MPT & Match between Person and Technology \\
& (Predisposition Assessment) \\
OT-FACT & Functional Performance \\
PIADS & Psycho-Social Impact \\
QUEST & Users' Satisfaction \\
SCAI & Costs \\
\hline
\end{tabular}

tion of a variety of AT devices. Seven of the instruments were designed to assess part of the overall outcome of AT and one instrument was designed to assess costs. For each of these instruments due attention has been given to the soundness of the instruments during the development. There are a few other instruments (e.g., COPM, Law et al. [2]) that might have been included in this special issue. These instruments were not included for practical reasons and their absence does not imply that the requirements were not met. The included instruments and the concepts they address are given in Table 1.

\section{Future challenges}

Although much effort has been invested in the instruments presented in this special issue, the field of assistive technology outcome assessment is still developing and faces more challenges: a) existing instruments must be further developed to improve quality and applicability, b) instrument application must be standardised, and c) international collaboration should be strengthened to improve both development and application of instruments.

a) Further development of instruments

Although the instruments included in this issue were designed as generic measures with respect to type of AT, its user and his or her social background, this requirement may not been met in all circumstances. Widespread application of each of the instruments in different conditions will reveal the extend to which the instruments are indeed generic and whether further improvements are required. International developments such as the update from ICIDH to ICF may also require adjustment of instruments. In support of the (further) development of instruments there should be a development of a framework ordering the elements of AT outcome. This could serve as a guideline and point of reference. b) Standardisation of instrument application

The application of instruments should be in its original format, the latest version should be used and instruments should not be further decomposed than intended. Furthermore, application must be performed according to their guidelines. Violation of this standardised administration leads to uncertainty about the reliability and validity and therewith about the quality of the resulting data. Data resulting from standardised application on the other hand could yield a number of positive effects such as increased quality and appreciation of the outcome study, increased comparability of outcome data leading to increased comparability between effectiveness data of types of AT, and finally, the facilitation of further development of the instrument or the illustration of the need for the development of a new instrument.

The need for standardisation of instrument application also involves the harmonisation of translation procedures concerning not only the language but also the societal background of AT use and provision.

c) International collaboration

The developments of instruments in different countries have been relatively independent. Most new initiatives to develop instruments in this field came from individual researchers and institutes who felt the need for specific AT oriented instruments. The result of this fragmented development is a number of promising instruments, most of which are presented in this special issue. The independent developments, however, also imply that the speed of the development is low and that the continuity of the development, dissemination and implementation of instruments is not certain. The quality of outcome measures for assistive technology would greatly benefit from a more coordinated international approach regarding development, dissemination and implementation. Developers in from North America and Europe could learn much from each other and developments would possibly go much faster and with higher quality.

With this special issue we hope to provide a starting point for these developments and to contribute in general to the enhancement of quality of assistive technology outcome assessment. 


\section{References}

[1] M.J. Fuhrer, Assistive technology outcomes research: challenges met and yet unmet, American Journal of Physical Medicine and Rehabilitation 80 (2001), 528-535.

[2] M. Law, S. Baptiste, A. Carswell, M. McColl, H. Polatajko and N. Pollock, Canadian Occupational Performance Measure, (3rd ed.), Ottawa, Ontario: CAOT, 1998.

[3] NIDDR (National Institute on Disability and Rehabilitation Research), Final Funding Priorities for Fiscal Years 2001-2003 for Four Disability and Rehabilitation Research Projects, Federal register 66(123) (June 26, 2001), 34025-34034. 